\title{
Gradhiva
}

GRADHIV

Revue d'anthropologie et d'histoire des arts

$14 \mid 2011$

Carl Einstein et les primitivismes

\section{Le Blaue Reiter selon Carl Einstein : lecture comparée (1926-1931)}

Introduction au Cavalier bleu

\section{Maria Stavrinaki}

\section{OpenEdition}

\section{Journals}

Édition électronique

URL : http://journals.openedition.org/gradhiva/2205

DOI : $10.4000 /$ gradhiva.2205

ISSN : 1760-849X

Éditeur

Musée du quai Branly Jacques Chirac

Édition imprimée

Date de publication : 30 novembre 2011

Pagination : 198-203

ISBN : 978-2-35744-046-3

ISSN : 0764-8928

Référence électronique

Maria Stavrinaki, «Le Blaue Reiter selon Carl Einstein : lecture comparée (1926-1931) », Gradhiva [En

ligne], 14 | 2011, mis en ligne le 30 mai 2012, consulté le 01 mai 2019. URL : http://

journals.openedition.org/gradhiva/2205 ; DOI : 10.4000/gradhiva.2205

(c) musée du quai Branly 


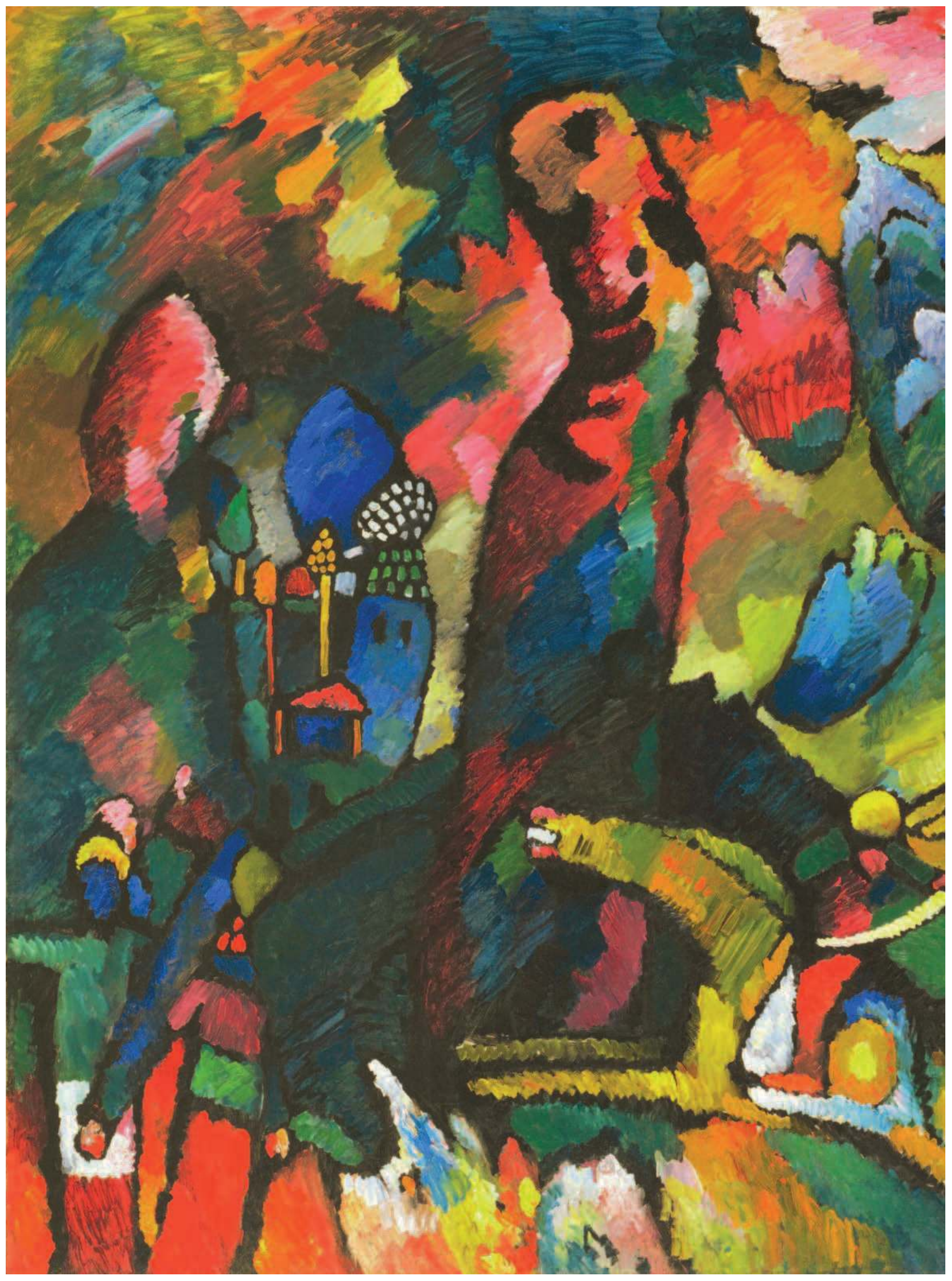




\title{
1931 Le Cavalier bleu
}

\section{Le Blaue Reiter selon Carl Einstein : lecture comparée [1926-1931]}

\author{
Introduction par Maria Stavrinaki
}

\author{
"Il existe deux montagnes sur lesquelles règnent la lumière \\ et la clarté, la montagne des animaux et la montagne des dieux. \\ Mais au milieu s'étend la crépusculaire vallée des hommes. \\ Quand il arrive à l'un d'eux d'élever là-haut ses regards, \\ il éprouve avec pressentiment, lui qui se sait ne pas savoir, \\ une insatiable nostalgie de ces êtres qui ne savent pas ne \\ pas savoir et de ces êtres qui se savent savoir.» \\ Paul Klee, Journal ${ }^{1}$ (1903)
}

\section{Le futur passé}

Carl Einstein a donné trois versions successives de son ouvrage Die Kunst des 20. Jahrhunderts (L'Art du xx siècle) dans un bref espace de cinq ans : 1926, 1928 et $1931^{2}$. Si, en s'impliquant dans ce projet éditorial consacré à l'art de son temps, Einstein témoignait de son attachement au présent, il n'exprimait pas moins sa volonté de soustraire l'art au flux de ce présent. Il traitait ce dernier avec la gravité qui était ordinairement réservée au passé noble et distant. Mais dans le même temps, il se propulsait dans le futur, afin d'interroger le potentiel utopique contenu dans l'art de son temps. Ainsi, même si son livre ne couvrait que les trois premières décennies $d u x^{e}$ siècle, son titre signifiait, avec aplomb, les nombreuses années à venir. Le régime oxymore du temps qu'Einstein investissait en tant qu'historien était finalement celui d'un «futur passé ». Mais ce futur passé était bien différent de celui théorisé plus tard par Reinhart Koselleck, aux prises avec une histoire en éternelle transition parce qu'en éternelle attente 3 . Si Einstein préjugeait du futur et le traitait comme s'il était déjà passé, c'est parce qu'il s'efforçait d'évaluer les expériences artistiques de son temps comme autant de défaites avérées, de victoires momentanées ou de promesses dans cette lutte du «sujet » et de l'«objet» qu'était, selon lui, l'histoire : «L'histoire de toute la philosophie, c'est l'histoire de la bataille entre le moi et l'objet, et l'homme, pour maintenir un rien de liberté et pour sortir d'un déterminisme fatal, est forcé à anéantir le monde donné par ses forces imaginatives." La bipolarité du "tectonique» et de l'«hallucinatoire» qu'il avait conçue pour comprendre les productions artistiques de l'homme était ainsi traduite en principe épistémologique de l'écriture de l'histoire de l'art de son temps. Il s'efforçait de discerner la "tectonique" qui agençait les «hallucinations » de son époque, et qui étaient en grande partie aussi les siennes.

La lutte des artistes contre le monde réifié avait commencé avec l'impressionnisme, auquel Einstein consacrait sa préface. Véritable mouvement charnière, l'impressionnisme était pour lui plus ambivalent qu'il ne l'avait été pour la plupart des avant-gardes, qui ont reconnu en lui leur contre-modèle. Sa temporalité instantanéiste et la dissolution formelle qui en dérivait n'étaient pas seulement les signes de l'atomisation, de l'équivocité et de la temporalisation modernes, mais aussi les premières affirmations de la volonté du sujet de revendiquer sa liberté face au monde réifié. Le regard du peintre impressionniste désagrégeait l'objet jusqu'à ses derniers atomes, mais ce regard était conçu comme un dispositif purement physiologique. Parce que la 


\section{TEXTES DE CARL EINSTEIN}

désagrégation impressionniste était matérialiste, elle conduisait à une équivalence parfaite entre l'homme et les choses, réduits à des touches pareillement minuscules, rapides et éphémères. Dès lors, le défi auquel a dû répondre L'Art du $\mathrm{XX}^{\mathrm{e}}$ siècle était de poursuivre la lutte contre l'objet sclérosé, mais en tentant de donner à la dissolution une charpente et, par là même, une assise métaphysique.

Dans les trois versions, le texte comptait plus de deux cents pages denses et plus de 600 reproductions. Leur comparaison permet d'observer les glissements et même les sauts conceptuels qui s'opèrent chez Einstein au fil de ces quelques années : L'Art du xx siècle est un palimpseste sur lequel bien des mutations ou des hésitations de l'auteur, qui était aussi une figure caractéristique de son temps, ont pu laisser leur empreinte. À ce titre, la partie sur Paul Klee, dont nous publions ici la première traduction en français, est un exemple très caractéristique. Dans les versions de 1926 et de 1928, Einstein ne consacrait pas un chapitre séparé au Blaue Reiter. Il incluait Franz Marc, Vassily Kandinsky et Klee au sein de la vaste section intitulée tout simplement "Les Allemands ». Mais, en 1931, il se décida à les traiter à part. Ce choix résulte de sa rencontre avec le surréa- lisme français, auquel il consacrait dans cette même version de son livre un chapitre intitulé «La génération romantique ». Le Blaue Reiter était pour luila génération romantique allemande ${ }^{5}$. Cette bifurcation nationale $d u$ romantisme contemporain ne visait qu'accessoirement à une symétrie entre la France et l'Allemagne. Elle était plutôt le résultat de l'écriture de l'histoire à partir du présent, principe épistémologique revendiqué souvent par Einstein. La relecture du Blaue Reiter à travers la grille surréaliste se manifestait concrètement par l'ajout d'une introduction, ainsi que de quelques passages nouveaux, où prédominaient des concepts clés pour l'Einstein de ces années-là : la "métamorphose", le «training», l'« inconscient » et d'autres.

L'appartenance de Klee au chapitre sur le Blaue Reiter n'était pas seulement légitime d'un point de vue factuel ${ }^{6}$. La mise en tension par Einstein de deux démarches différentes - celle des deux initiateurs de l'entreprise, Kandinsky et Marc, et celle de Klee - était productrice de sens. Einstein suivait à son insu la méthode choisie par Klee lui-même, qui avait esquissé dans son journal sa position ontologique et la conception de l'art qu'il en tirait en se comparant à Marc, ami proche qui venait de mourir au front : « Quand je dis qui
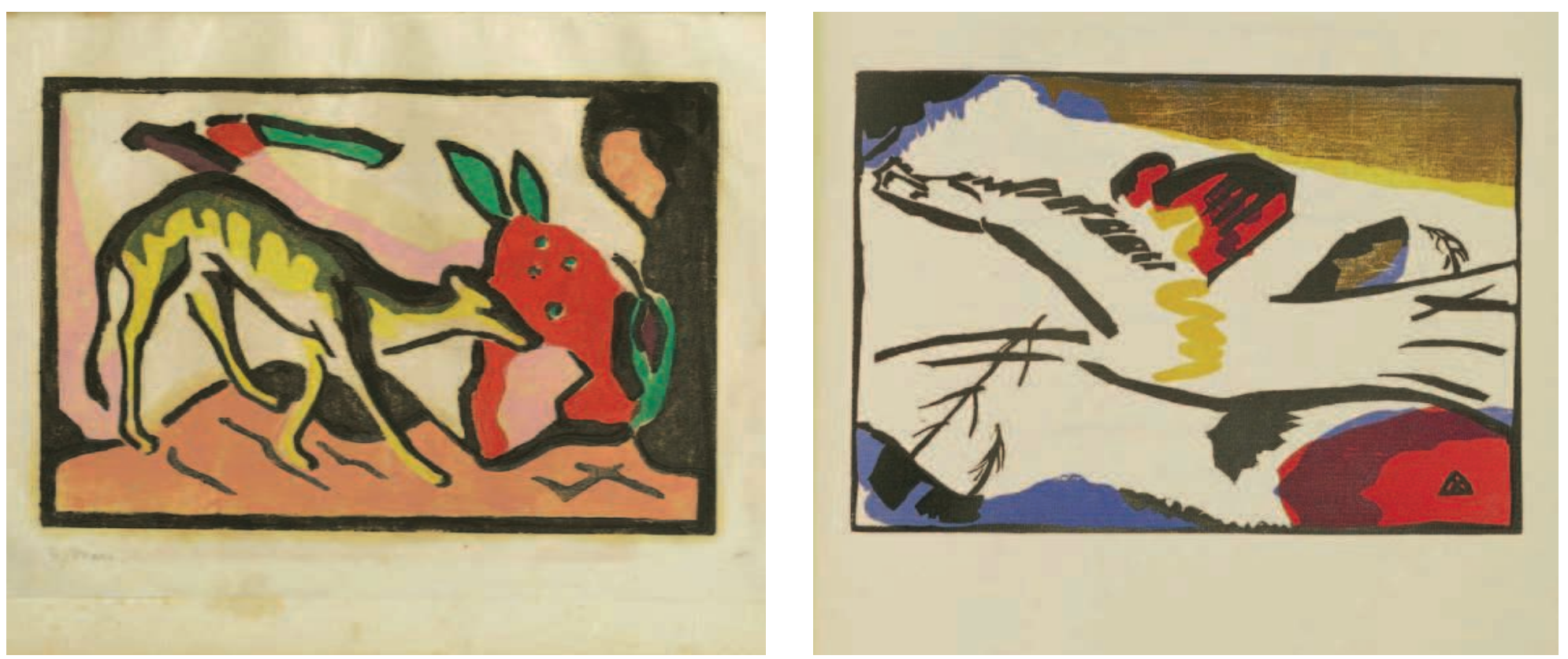

Fig. 2, à gauche, Vassily Kandinsky, Lyrical (planche, folio 9) in Klaenge ("Sons »), 1913, MoMA, New York / Scala, Florence @ ADAGP, Paris 2011. À droite, Franz Marc, Fabeltier ("Créature fantastique ") in Der Blaue Reiter, 1912. MoMA, New York / Scala, Florence.

Fig. 1 Vassily Kandinsky, Peinture avec un archer, 1909, MoMA, New York, don et legs de Mme Bertram Smith 1959. The Museum of Modern Art, New York / Scala, Florence @ ADAGP, Paris 2011. 
est Franz Marc, je dois confesser en même temps qui je suis moi-même, car bien des choses qui me concernent lui appartiennent aussi7. » Ce partage de «bien des choses » était dessiné par Klee dans une lettre envoyée à Marc en 1915. Noircissant la partie où deux cercles se croisaient, il expliquait : «Je place bien sûr le Moi divin au centre [c'est l'auteur qui souligne]. Ce moi est à mes yeux le seul fiable et ma confiance dans les autres ne repose que sur ce qu'il y a de commun. Ton cercle et mon cercle m'ont paru posséder une partie commune relativement importante; quelque chose comme ça $\left[\right.$ dessin ${ }^{8}$ ]. » Cet « entremonde » (Zwischenwelt), formé par deux moi apparentés qui ne pouvaient partager «bien des choses " qu'à condition de ne jamais coïncider, était le modèle structurel à travers lequel Klee pensait presque tout dans l'art et dans la vie : les rapports entre le sujet et l'objet, entre la terre et l'univers, entre l'espace et le temps. Surtout, c'était la surface picturale elle-même qui était conçue et fabriquée comme un Zwischenwelt, comme la partie commune, mais provisoire, du sujet et $\mathrm{du}$ monde, du fond et de la figure, du devenir et de la forme. Cette idée d'«entremonde » séparait Klee de son ami Marc. Et cela n'avait pas échappé à Einstein.

\section{Le «pont » et l'«entremonde »}

Pour Einstein, les deux pôles entre lesquels l'art moderne menait son combat étaient celui de la pure subjectivité, émancipée de toute référence au réel, et celui de la réalité réifiée. Rien ne pouvait changer l'opinion qu'il se faisait de l'art de Kandinsky et de Marc: de 1926 à 1931, les deux artistes étaient restés fixés au pôle subjectif de l'art ${ }^{9}$. Einstein expliquait l'abstraction plus ou moins poussée de leurs formes comme la simple conséquence de l'abstraction ontologique qu'impliquait leur volonté de fuir le réel afin de s'identifier avec les seules émanations de leur esprit. "L'empathie est l'abstraction " : on peut transposer aussi parfaitement la formule élaborée par Peter Szondi pour expliquer le « naïf » etle « sentimental » chez Friedrich von Schiller ${ }^{10}$. Cette conception dialectique des deux notions a priori opposées dans l'esprit de Wilhelm Worringer signifiait le besoin positif, chez ces deux artistes, de s'identifier à l'esprit aux dépens d'une quelconque volonté négative de supprimer le réel ${ }^{11}$. Einstein avait bien vu cette différence, qui allait être vite oubliée du reste par les historiens de l'art plus tardifs, toujours prêts à lier le Blaue Reiter au Kunstwollen (volonté d'art) abstrait. Là où Einstein se trompait cependant, c'était dans son choix de traiter Kandinsky et Marc de manière identique. Même si Marc n'a fait que frôler l'abstraction, sa conception de l'art était beaucoup plus radicale que celle de Kandinsky. Ce dernier, plus goethéen au fond, savait pertinemment que l'esprit avait besoin de la forme pour agir. Passionnément " artiste », Kandinsky ne niait pas la matière, qui était la condition même de son art. Il en fut autrement pour Marc, qui donnait de celui-ci la définition suivante : "La nature est partout, en nous et en dehors de nous; il y a une seule chose qui n'est pas entièrement nature mais plutôt victoire sur elle et interprétation; sa force prend naissance en un point qui nous est inconnu : c'est l'art. L'art a toujours été et est dans son essence l'éloignement le plus téméraire de la nature et du "naturel" qui ait jamais existé; il est le pont vers le royaume de l'esprit, la nécromancie de l'humanité12. " Le "pont " que Marc jetait par l'art vers «le royaume de l'esprit » était à sens unique : il lui était de plus en plus difficile de rétablir la distance qu'il avait avec le motif avant son identification à celui-ci. En définissant la peinture comme "pont ", Marc lui ôtait toute capacité de présenter l'absolu, auquel pourtant il aspirait lui-même avec ardeur. Il voulait déchirer le voile de la représentation, mais son idéalisme radical lui interdisait de voir dans la peinture davantage qu'une fausse apparence. Ainsi, ce n'est pas un hasard s'il déclara avoir trouvé la "vérité de la peinture " une fois au front, loin de ses toiles et de ses pinceaux. Ses lettres et ses textes témoignent d'un repli sur soi et d'une extinction du monde "en flammes " de plus en plus sévères. Détaché de ses semblables et de l'histoire, Marc sombrait dans ce qu'Einstein appelait tantôt le «monologue » et tantôt l'« autisme».

Quant à Klee, il chercha toujours à confirmer par son art la possibilité d'une peinture polyphonique et contrapunctique. Par suite, le champ qu'il occupa sans interruption dans L'Art du $x x^{\mathrm{e}}$ siècle fut celui du Zwischenwelt où se croisaient sujet et objet, hallucination et mémoire, abstraction et figuration. Mais, en 1931, Einstein attribuait une valeur bien différente à cet espace intermédiaire que dans son texte de 1926, resté inchangé encore en 1928.

Einstein écrivait alors : « Klee a emprunté une autre voie; il en reste au motif et met à la place des objets donnés des contenus inventés, issus de la liaison de l'image-souvenir [Erinnerungsbild] et de l'hallucinatoire[Halluzinativ]; Klee n'isole pas les éléments visionnaires, mais les intègre dans ce qui a été vu, il utilise l'opposition et la force métamorphique des deux [...]. Klee observe avec un humour subtil sa propre vision, 


\section{TEXTES DE CARL EINSTEIN}

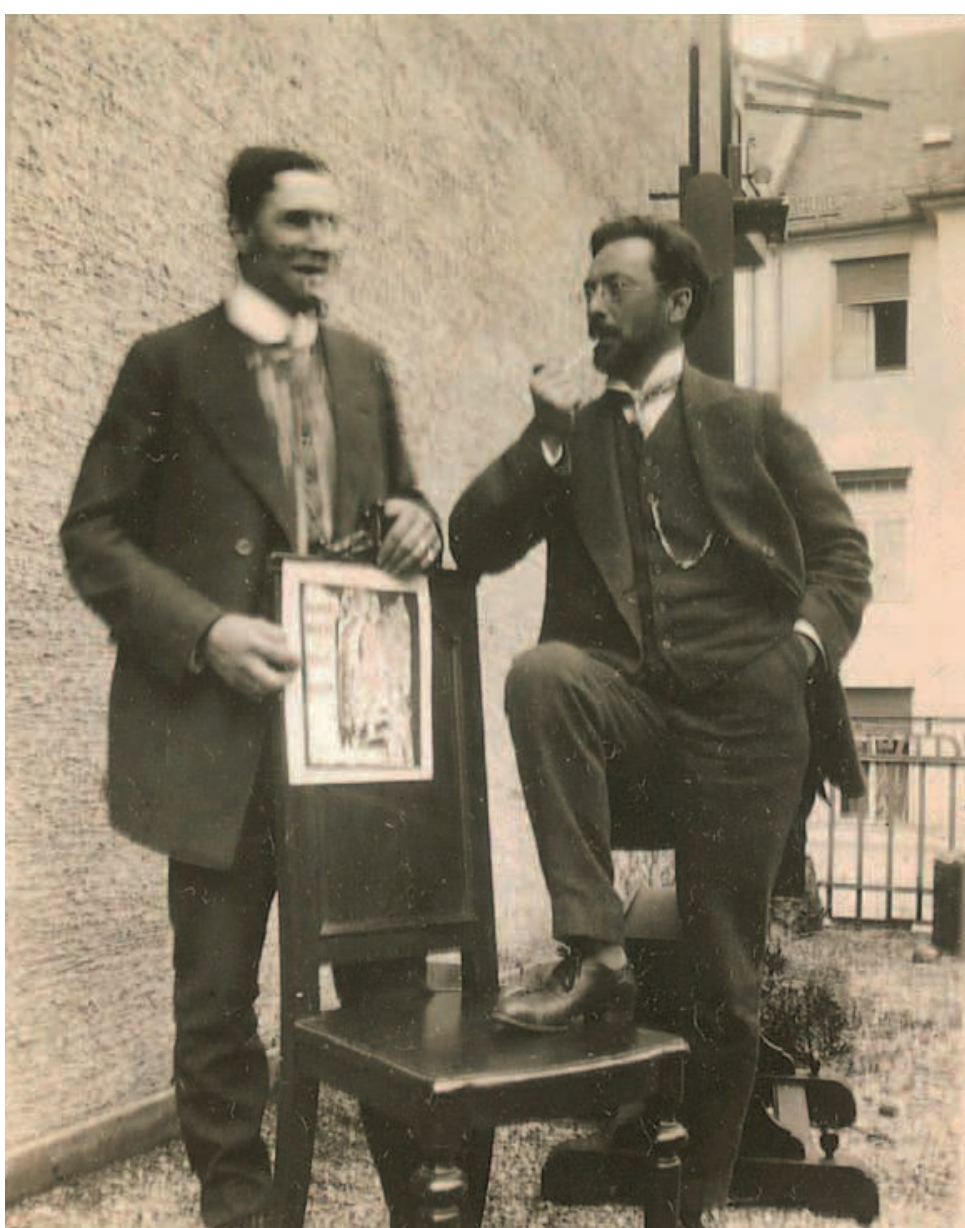

Fig. 3 Franz Marc et Vassily Kandinsky avec la xylographie de l'almanach Der Blaue Reiter sur le balcon du 36 Ainmillerstrasse, Munich, 1911-1912, collection Gabriele Münter et Johannes Eichner, Fondation de Munich.

il ne prend pas seulement ses distances par rapport au donné, mais fuit les images, métaphysiquement et de manière un peu railleuse, toujours prêt à voir ses propres visages avec un humour moqueur ${ }^{13}$. " L'entremonde de Klee était donc celui de l'humour, de l'ironie, du grotesque - exigeant ainsi nécessairement l'action conjointe de deux forces antithétiques : celle des souvenirs, agents de la conservation du passé, et celle des hallucinations, matrices potentielles du futur. Mais, aux yeux d'Einstein, cette action contrastée qui avait engendré et mis en forme le Zwischenwelt de Klee restait fondamentalement ambiguë.

Bien sûr, à la différence d'un Marc qui cherchait l'empathie inconditionnelle avec les animaux que son esprit engendrait à volonté, Klee observait un détachement égal vis-à-vis de ses hallucinations et du réel. Des figures d'un rêve concret en résultaient. Mais si Klee réussissait à rompre ainsi le cercle vicieux du moi, rien ne garantissait, selon Einstein, la viabilité et l'efficacité de l'action de ses rêves concrets en dehors de la zone étroite de son Zwischenwelt : «La vengeance de la nature repose sur la minceur de toute subjectivité, dont la confirmation dépend presque de l'humeur du spectateur ${ }^{14}$. " Ce qui faisait défaut à l'artiste, c'était la force univoque du mythe, qui sait s'imposer aveuglément et impérativement, brisant cette accumulation des habitudes qui fait la constitution biologique du réel : "Klee part à la découverte d'une nouvelle réalité. Nous ne savons pas dans quelle mesure elle sera assez puissante pour s'affirmer dans le monde biologique habituel, dans quelle mesure elle possède la force nécessaire pour obliger l'objectivité. [...] Klee crée un petit Zwischenwelt qui ne sera certes pas élevé au rang d'un cas d'école, mais dont la piété lunatique ne doit pas être refusée. Parce qu'un domaine symbolique objectif nous fait encore défaut, il se crée sa mythologie privée ${ }^{15}$. " Einstein doutait que les êtres grotesques de Klee puissent respirer dans l'espace réel, que leur force visuelle soit assez déterminée pour interrompre les automatismes accumulés et la continuité biologique. Tout cela revenait à dire que l'ambiguïté du Zwischenwelt consistait dans sa fertilité incertaine. Les formes intermédiaires qui habitaient les tableaux de Klee étaient les produits mixtes du «raffinement» de la couleur et du dessin "primitif ${ }^{16}$ ". Tard venues et primitives, femmes et mâles à la fois, les grotesques couraient le risque de rester fixées au présent du Zwischenwelt.

En 1931, cependant, le scepticisme d'Einstein semblait considérablement guéri, grâce, notamment, aux ressorts révolutionnaires objectifs qu'il avait détectés, à l'instar de plusieurs de ses contemporains, au sein de la subjectivité. Le changement se faisait remarquer d'entrée de jeu lorsqu'il évoquait «les couches plus lointaines, plus décisives" dans lesquelles pénétrait la vision de Klee comparativement à celle de Kandinsky. C'est que les "couches" et les "strates" de l'inconscient étaient devenues entretemps un gage d'objectivité précieux pour tous ces intellectuels qui cherchaient à affirmer la possibilité d'une révolution menée en dehors d'une conception strictement matérialiste de l'histoire. La temporalité "grotesque» de l'art de Klee en 1926 et en 1928, paralysée entre le "trop tard" et le "pas encore", se transformait en 1931 en une synchronie de l'archaïque et du nouveau. Cette "non-contemporanéité » détectée au sein même du «contemporain » était détentrice de toute la force alogique qu'Einstein jugeait nécessaire à la métamor- 
phose de l'histoire. «Héritages oubliés » et «forces non adaptées ", que l'identité biologique n'avait pas su s'assimiler encore, devenaient les agents d'une "discordance avec le monde environnant ». L'archaïque était précieux pour Einstein : il lui offrait aussi bien un pont entre le collectif et le subjectif que la possibilité d'une révolution réfractaire à la notion de progrès. La théorie de la récapitulation, postulant une analogie structurelle entre l'évolution de l'individu et celle de l'espèce, constatait une porosité entre leurs acquis respectifs. Bien que, en 1931, le monde symbolique commun ne fût toujours pas objectivé, il était réconfortant de penser que chaque personne avait pu en avoir l'avant-goût lors de son enfance, âge de la synchronie parfaite, où la récapitulation des ancêtres coïncidait avec l'absolue nouveauté de la sortie dans le monde.

Tout porte à croire que l'air avait bel et bien changé dans le Zwischenwelt de Klee et que les créatures qui le peuplaient se voyaient reconnaître maintenant une certaine viabilité. Si cet entremonde continuait à être formé par l'action conjointe de deux forces antithétiques, la fertilité de leur action dialectique ne semblait plus faire de doute. Sous l'influence de l'ethnologie, qu'il approfondissait de plus en plus, et du surréalisme dans ses différentes versions, Einstein présentait l'expression du principe du nouveau et de l'hallucinatoire comme une "transe", une "écriture médiumnique", un "psychogramme». Mais l'«image-souvenir» disparaissait de son développement au profit du «tectonique", qui était le médiateur des ancêtres et de leurs cultures collectives dans le monde des formes. Cette substitution entraînait immédiatement la fin du travail de sape de l'ironie au profit du travail créateur de la "métamorphose ». Selon Einstein, les formes continuaient à être intermédiaires et mixtes dans les œuvres de Klee, mais leur caractère grotesque était présenté désormais comme une identité proprement métamorphique, issue de la synergie du végétal et du minéral, de l'hallucinatoire et du tectonique, du surréalisme et du cubisme. L'identité métamorphique devenait le paradigme fonctionnel de la subjectivité, brisant la mêmeté biologique et l'univocité des objets.

Tel fut le renversement qui conditionnait la pensée d'Einstein : si le passé atavique était pour les primitifs un principe conservateur, les obligeant à respecter les esprits des morts et à défendre l'ordre social établi, il se convertissait, chez les modernes, en un principe révolutionnaire. En médiatisant le mythe objectif qui leur faisait défaut, il les protégeait des excès de leur subjectivité, qui demeurait le foyer de la révolution ${ }^{17}$.

\section{-}

1. Paul Klee, Journal, trad. Pierre Klossowski, Paris, Grasset, 1959, p. 152 ; Tagebücher 1898-1918 (nouvelle édition critique), Wolfgang Kersten (éd.), Berne, Paul Klee-Stiftung-Stuttgart, Gerd Hatje Verlag, 1988, § 539, p. 180.

2. L'ouvrage faisait partie de la prestigieuse collection «Kunstgeschichte 》 (《Histoire de l'art ») des éditions Propyläen, consacrée à l'histoire universelle de l'art.

3. Cf. Reinhart Koselleck, Le Futur passé. Contribution à la sémantique des temps historiques, Paris, EHESS, 1990.

4. Carl Einstein, texte sans titre [il se pose la question] inédit, rédigé en français, destiné vraisemblablement à une conférence, vers 1928, Werke, t. IV, Hermann Haarmann et Klaus Siebenhaar (éd.), Berlin, Fannei \& Walz, 1994, p. 182.

5. Einstein écrivait ainsi à propos de Klee dans l'introduction qu'il ajoutait au chapitre en question en 1931 : «Klee, quant à lui, a abandonné les sphères bornées de l'indifférence esthétique et s'est lancé courageusement dans la réalisation de formes. Voilà pourquoi nous voyons en lui le représentant majeur du nouveau romantisme allemand. »; Carl Einstein, Die Kunst des 20. Jahrhunderts, Berlin, Propyläen Verlag, 1931, p. 195.

6. Paul Klee avait participé aux expositions du groupe.

7. Paul Klee traduit dans Maria Stavrinaki (éd.), Franz Marc, Écrits et correspondances, trad. Thomas de Kayser, Paris, Ensb-a, 2006, p. 454.

8. Lettre de Paul Klee à Franz Marc, 8 juin 1915, in Maria Stavrinaki (éd.), op. cit., p. 445.

9. Une première traduction partielle, due à Liliane Meffre, du texte consacré à Kandinsky et à Marc avait paru dans Les Cahiers du musée national d'Art moderne 1, 1979, p. 32-38.

10. Peter Szondi, «Le naïf est le sentimental », Poésie et Poétique de l'idéalisme allemand, trad. Jean Bollack, Paris, Gallimard, 1975, p. 4793. Nous nous permettons de renvoyer à notre texte « L'empathie est l'abstraction : réflexions sur l'art et la vie de Franz Marc », Pratiques. Réflexions sur l'art 16, Presses Universitaires de Rennes, 2005, p. 26-63.

11. Seul le gothique était, pour Worringer, une volonté de forme réconciliant l'empathie et l'abstraction.

12. Franz Marc, «La nouvelle peinture » (1912), in Maria Stavrinaki (éd.), op. cit., p. 150.

13. Carl Einstein, Die Kunst des 20. Jahrhunderts, Berlin, Propyläen Verlag, 1926, p. 154.

14. Ibid., p. 155.

15. Ibid.

16. Klee expliquait l'« impression primitive » qui pouvait émaner parfois de son œuvre comme une «économie et, par là, la connaissance professionnelle ultime. Elle est donc l'opposé de la primitivité véritable »; Paul Klee (1909), Tagebücher, op. cit., § 857, p. 294.

17. Sur l'interprétation de l'art de Paul Klee par Einstein, cf. Uwe Fleckner, Carl Einstein und sein Jahrhundert. Fragmente einer intellektuellen Biographie, Berlin, Akademie Verlag, 2006, surtout p. 385-390; id., The Invention of the 20th Century. Carl Einstein and the Avant-Gardes, Madrid, Museo Nacional Centro de Arte Reina Sofia, 2009, p. 229-281; Sebastian Zeidler, «Form as Revolt », Res: Journal of Anthropology and Aesthetics 57-58, 2010, p. 229-263. 